TITLE:

\title{
Entropic insertion of a big sphere into a cylindrical vessel
}

$\operatorname{AUTHOR}(S):$

Amano, Ken-ich; Kinoshita, Masahiro

CITATION:

Amano, Ken-ich ... [et al]. Entropic insertion of a big sphere into a

cylindrical vessel. Chemical Physics Letters 2010, 488(1-3): 1-6

ISSUE DATE:

2010-03-12

URL:

http://hdl.handle.net/2433/120742

\section{RIGHT:}

(c) 2010 Elsevier B.V.; この論文は出版社版でありません。引用の際には 出版社版をご確認ご利用ください。; This is not the published version. Please cite only the published version. 


\title{
Entropic Insertion of a Big Sphere into a Cylindrical Vessel
}

\author{
Ken-ich Amano ${ }^{1}$ and Masahiro Kinoshita ${ }^{2}$ \\ ${ }^{1}$ Graduate School of Energy Science, Kyoto University, Uji, Kyoto 611-0011, Japan \\ ${ }^{2}$ Institute of Advanced Energy, Kyoto University, Uji, Kyoto 611-0011, Japan
}

Author to whom correspondence should be addressed: Masahiro Kinoshita.

E-mail address: kinoshit@iae.kyoto-u.ac.jp.

\begin{abstract}
We calculate the spatial distribution of the entropic potential between a big sphere and an even bigger vessel with cylindrical shape, which are immersed in small spheres, using the three-dimensional integral equation theory. The distribution is strongly dependent on relative magnitudes of the big-sphere diameter and the inner diameter of the vessel unless the latter is much larger than the former. For a fixed value of the inner diameter, a big sphere whose diameter lies in a specific range is spontaneously inserted into the vessel and strongly confined within a small space almost in the center of the vessel cavity.
\end{abstract}




\section{Introduction}

The entropic excluded-volume effect plays crucially important roles not only in colloidal suspensions but also in biological systems [1-3]. Many biological processes are controlled by the interactions between macromolecules and by those of macromolecules with cell membranes. The macromolecules and membranes generate excluded volumes for smaller particles forming the solvent (i.e., volumes of the spaces which the centers of solvent particles cannot enter). When the macromolecules approach each other, for instance, the excluded volumes overlap, leading to an increase in the total volume available to the translational displacement of solvent particles. That is, the number of accessible configurations of the solvent increases and a corresponding entropy gain occurs. Thus, an attractive force is induced between macromolecules at small separations $[4,5]$. The entropic forces are largely influenced by the overall shape of macromolecules and membranes as well as their detailed polyatomic structures. The entropic effect is omnipresent and substantially large. In statistical mechanics, the force and the interaction are described by the mean force and by the potential of mean force, respectively.

To elucidate the entropic effect, theoretical studies dealing with big bodies with simple geometries immersed in small particles have been performed rather extensively. In these studies, the hard-body models are employed for the big bodies and small particles. In the hard-body models, all the accessible system configurations share the same energy, and the system behavior is purely entropic in origin: They allow us to investigate the entropic effect exclusively. In biological systems, the solvent is water characterized by hydrogen bonds. However, in the entropic gain upon the solute contact and related processes, the translational entropy predominates over the rotational entropy. The entropic effect considered here can be analyzed by modeling water as hard spheres in many cases, as long as the diameter and number density are set at the values of water $[2,3]$. A more detailed argument is given as the last paragraph in Sec. 3.2.

The most fundamental subject first considered is the entropic force or interaction induced between big spheres or between a big sphere and a flat wall immersed in small spheres [6-11]. It has been extended to more advanced subjects, and a significant amount of interesting information is now available. For example, the entropic interaction between a big sphere and a concave surface is stronger than that between the same big sphere and a flat surface [12]. For a big sphere on a wall, the geometric features of the wall induce entropic force acting on the big sphere in a specific direction along the wall [13-15]: The big sphere is locally repelled from a step edge, attracted to a corner, and moved from a convex surface to a concave one. The entropic torque induces the rotation of a big body with asphericity [16], the entropic interaction between big aspherical bodies leads to their ordered aggregation $[17,18]$, and the lock-key interaction is entropically provided 
with high size selectivity $[13,14,19]$. Recently, the interaction between big spheres in a multi-component mixture of small spheres has been analyzed [20].

Elaborate statistical-mechanical methods such as the density functional theory [12] and the integral equation theory $[6,11,13,14]$ are employed in the studies on the entropic effect described above. An important feature revealed is the following. The effect of the density structure of small spheres near the big bodies is essential, making the entropic interaction rather complicated: It exhibits a great variation with the period which is close to the diameter of small spheres $d_{\mathrm{S}}$, and attractive and repulsive regions appear alternately. For the interaction between big spheres or between a big sphere and a flat wall, for instance, a strong repulsive peak appears at $h \sim 0.5 d_{\mathrm{S}}$ where $h$ is the surface separation [1,2,6-11]. The details of the entropic effect are strongly dependent on the geometric characteristics of the system considered. These properties cannot be reproduced by the simple Asakura-Oosawa theory $[4,5]$ which gives only a much shorter-ranged, monotonically changing attractive force. It is challenging to investigate the entropic effect for a new system using an elaborate statistical-mechanical theory.

Here we consider the insertion of a big body into an even bigger vessel in small spheres forming the solvent. Such an insertion is a basic process in biological systems. Typical examples are the introduction of a polypeptide into the chaperonin GroEL [21,22] and that of an antibiotic molecule or a toxic protein into a cell-membrane protein $[23,24]$. They form elementary steps in the facilitation of protein folding and in the export of unfavorable molecules to the outside, respectively. In the present study, a simplified model system is treated to explore the roles of the entropic effect as an important first step. We calculate the spatial distribution of the entropic potential between a big sphere and an even bigger vessel with cylindrical shape (see Fig. 1), which are immersed in small spheres. As in the earlier works, the hard-body models are employed for the big sphere, vessel, and small spheres. The vessel has length $L$, inner diameter $d_{\mathrm{I}}$, and outer diameter $d_{\mathrm{O}}$. The big sphere has diameter $d_{\mathrm{B}}\left(d_{\mathrm{B}} \leq d_{\mathrm{I}}\right)$. The vessel and the big sphere are immersed in small spheres with diameter $d_{\mathrm{S}} . L,\left(d_{\mathrm{O}}-d_{\mathrm{I}}\right) / 2$, and $d_{\mathrm{B}}$ are fixed at $7 d_{\mathrm{S}}, 6 d_{\mathrm{S}}$, and $5 d_{\mathrm{S}}$, respectively, and $d_{\mathrm{I}}$ is varied as an essential parameter. The three-dimensional (3D) integral equation theory $[13,14,17,25-29]$ is employed in the calculation.

It is shown that the spatial distribution of the entropic potential is remarkably dependent on $d_{\mathrm{I}}$ unless $d_{\mathrm{I}}$ is much larger than $d_{\mathrm{B}}$. The behavior exhibited when $d_{\mathrm{B}}$ is varied with $d_{\mathrm{I}}$ kept constant can also be conjectured: For a fixed value of $d_{\mathrm{I}}$, a big sphere whose diameter lies in a specific range is not only spontaneously inserted into the vessel but also strongly confined within a small space almost in the center of the vessel cavity (i.e., separated from the inner surface). Moreover, the specific range becomes wider as $d_{\mathrm{B}}$ increases. 


\section{Theory}

It is assumed that solute $i$ of arbitrary geometry is immersed at infinite dilution in small spheres with diameter $d_{\mathrm{S}}$ forming the solvent. The Ornstein-Zernike (OZ) equation in the Fourier space $[13,14]$ is expressed by

$$
W_{i \mathrm{~S}}\left(k_{x}, k_{y}, k_{z}\right)=\rho_{\mathrm{S}} C_{i \mathrm{~S}}\left(k_{x}, k_{y}, k_{z}\right) H_{\mathrm{SS}}(k)
$$

and the hypernetted-chain (HNC) closure equation $[13,14]$ is written as

$c_{i \mathrm{~S}}(x, y, z)=\exp \left\{-u_{i \mathrm{~S}}(x, y, z) /\left(k_{\mathrm{B}} T\right)\right\} \exp \left\{w_{i \mathrm{~S}}(x, y, z)\right\}-w_{i \mathrm{~S}}(x, y, z)-1$.

Here, the subscript " $\mathrm{S}$ " denotes the solvent, $w=h-c, c$ is the direct correlation function, $h$ the total correlation function, $u$ the potential, $\rho$ the bulk density, $k_{\mathrm{B}}$ Boltzmann's constant, $T$ the absolute temperature. The values of $d_{\mathrm{S}}$ and $\rho_{\mathrm{S}} d_{\mathrm{S}}{ }^{3}$ are set at those of water at $298 \mathrm{~K}$ and $1 \mathrm{~atm}, 0.28 \mathrm{~nm}$ and 0.7317 , respectively. The capital letters $(C, H$, and $W)$ represent the Fourier transforms. $H_{\mathrm{SS}}(k)$ $\left(k^{2}=k_{x}{ }^{2}+k_{y}{ }^{2}+k_{z}^{2}\right)$ calculated using the radial-symmetric HNC theory for spherical particles is part of the input data. We emphasize that the OZ equation is exact [6] (its approximate aspect has been pointed out in a literature [15], but this is incorrect). On the other hand, the bridge function is neglected in the HNC closure equation. However, it has been verified that the 3D-OZ-HNC theory gives quantitatively reliable results $[13,14]$.

The numerical procedure is briefly summarized as follows: $(1) u_{i}(x, y, z)$ is calculated at each 3D grid point, (2) $w_{i \mathrm{~S}}(x, y, z)$ is initialized to zero, (3) $c_{i \mathrm{~S}}(x, y, z)$ is calculated from Eq. (2), and $c_{i \mathrm{~S}}(x, y, z)$ is transformed to $C_{i \mathrm{~S}}\left(k_{x}, k_{y}, k_{z}\right)$ using the 3D fast Fourier transform (3D-FFT), (4) $W_{i \mathrm{~S}}\left(k_{x}\right.$, $\left.k_{y}, k_{z}\right)$ is calculated from Eq. (1), and $W_{i S}\left(k_{x}, k_{y}, k_{z}\right)$ is inverted to $w_{i S}(x, y, z)$ using the 3D-FFT, and (5) steps (3) and (4) are repeated until the input and output functions for $w_{i \mathrm{~S}}(x, y, z)$ become identical within convergence tolerance. On grid points where a solvent particle and the solute overlap, $\exp \left\{-u_{i \mathrm{~S}}(x, y, z) /\left(k_{\mathrm{B}} T\right)\right\}$ is zero. On those where a solvent particle is in contact with the solute, it is set at 0.5 , and otherwise it is unity. The grid spacing ( $\Delta x, \Delta y$, and $\Delta z$ ) is set at $0.1 d_{\mathrm{S}}$, and the grid resolution $\left(N_{x} \times N_{y} \times N_{z}\right)$ is $512 \times 512 \times 512$. It has been verified that the spacing is sufficiently small and the box size $\left(N_{x} \Delta x, N_{y} \Delta y, N_{z} \Delta \mathrm{z}\right)$ is large enough for the correlation functions at the box surfaces to be essentially zero.

We consider solutes 1 and 2. Solute 1 is a big vessel with cylindrical shape illustrated in Fig. 1. Solute 2 is a big sphere with diameter $d_{\mathrm{B}}$. First, the solute 1-solvent correlation functions (the Fourier transform of the direct correlation function is denoted by $\left.C_{1 \mathrm{~S}}\left(k_{x}, k_{y}, k_{z}\right)\right)$ are calculated by 
following the procedure described above $(i=1)$. Second, the solute 2-solvent correlation functions (the Fourier transform of the total correlation function is denoted by $H_{2 \mathrm{~S}}(k)$ ) are calculated using the radial symmetric HNC theory for spherical particles. The entropic potential between solutes 1 and 2 is described by the potential of mean force by assuming that the solvent particles are always in equilibrium with each configuration of the two solutes.

The potential of mean force between the two solutes $\Phi_{12}(x, y, z)$ are then obtained from

$\Phi_{12}(x, y, z) /\left(k_{B} T\right)=u_{12}(x, y, z) /\left(k_{\mathrm{B}} T\right)-w_{12}(x, y, z)$

where $w_{12}(x, y, z)$ is calculated by inverting $W_{12}\left(k_{x}, k_{y}, k_{z}\right)$ given by

$W_{12}\left(k_{x}, k_{y}, k_{z}\right)=\rho_{\mathrm{S}} C_{1 \mathrm{~S}}\left(k_{x}, k_{y}, k_{z}\right) H_{2 \mathrm{~S}}(k)$.

The physical meaning of $\Phi_{12}(x, y, z)$ can be understood from

$\Phi_{12}(x, y, z)=F(x, y, z)-F(\infty, \infty, \infty)$

and

$g_{12}(x, y, z)=\exp \left\{-\Phi_{12}(x, y, z) /\left(k_{\mathrm{B}} T\right)\right\}, g_{12}(\infty, \infty, \infty)=1$

Here, the origin of the coordinate system is chosen as illustrated in Fig. $1, F(x, y, z)$ is the free energy of small spheres in the case where the big sphere is at the position $(x, y, z)$, and $g_{12}(x, y, z)$ the pair distribution function. Due to the hard-body models, the behavior of $\Phi_{12}(x, y, z)$ is purely entropic in origin. Hereafter, $\Phi_{12}(x, y, z)$ is referred to as the entropic potential. A great advantage of the $3 \mathrm{D}$ integral equation theory is that the values of $\Phi_{12}$ on all the grid points are obtained from only a single calculation, which is in marked contrast with the usual computer simulation.

\section{Results and Discussion}

\subsection{Effect of inner diameter of vessel}

The spatial distribution of the entropic potential (on the cross section $(z=0)$ ) scaled by $k_{\mathrm{B}} T$ between the big sphere and the vessel are shown in Figs. 2-4 for several different values of $d_{\mathrm{I}}$. In 
the case of $d_{\mathrm{l}}=d_{\mathrm{B}}$ (Fig. 2 (a)), a significant lowering of the free energy of small spheres occurs when the big sphere is inside the vessel. However, there is high free-energy barrier $\left(\geq 3 k_{\mathrm{B}} T\right.$ : the case of the lowest barrier is indicated by the thin solid arrow) for the big sphere to overcome, which hinders the big-sphere insertion into the vessel. It has been shown in an experimental study for the entropic interaction between large spheres immersed in small spheres [30] that a barrier which is significantly higher than $k_{\mathrm{B}} T$ can hardly be overcome.

When $d_{\mathrm{I}}$ is increased to $d_{\mathrm{B}}+d_{\mathrm{S}}$ (Fig. 2 (b)), the spatial distribution of the entropic potential exhibits a drastic change. The big sphere is largely destabilized (i.e., the free energy of small spheres becomes much higher) inside the vessel except at the positions in contact with the inner surface of the vessel: It is largely destabilized around the $x$-axis. Since there is high barrier $\left(\geq 3 k_{\mathrm{B}} T\right.$ : the case of the lowest barrier is indicated by the thin solid arrow) for the big sphere to overcome, the big sphere can reach the contact positions only with low probability.

For $d_{\mathrm{I}}=d_{\mathrm{B}}+2 d_{\mathrm{S}}$ (Fig. 3 (a)), a domain within which the big sphere is highly stabilized (i.e., the free energy of small spheres becomes much lower) appears around the $x$-axis. The lowest barrier indicated by the thin broken arrow is $\sim k_{\mathrm{B}} T$, and it is not difficult for the big sphere to reach the domain. Along the $x$-axis inside the vessel, the potential is oscillatory with large amplitude as shown in Fig. 5 (a). Due to sufficiently high barriers along the $x$-axis, the big sphere is confined within the small space around the position indicated by the thick solid arrow where the local potential minimum is $\sim-14 k_{\mathrm{B}} T$. As observed in Fig. 3 (a), the big sphere can contact the inner surface of the vessel only with low probability due to high barriers $\left(\geq 3 k_{\mathrm{B}} T\right.$ : the case of the lowest barrier is indicated by the thin solid arrow).

When $d_{\mathrm{I}}$ is increased to $d_{\mathrm{B}}+3 d_{\mathrm{S}}$ (Fig. $3(\mathrm{~b})$ ), there appear two domains within which the big sphere is stabilized between the inner surface and the $x$-axis. The lowest barrier indicated by the thin broken arrow is $\sim k_{\mathrm{B}} T$, and it is not difficult for the big sphere to reach the domains. The big sphere is confined within the small space around the position indicated by the thick solid arrow where the local potential minimum is $\sim-6 k_{\mathrm{B}} T$. The big sphere can contact the inner surface of the vessel only with low probability due to high barriers ( $\geq 3 k_{\mathrm{B}} T$ : the case of the lowest barrier is indicated by the thin solid arrow).

When $d_{\mathrm{I}}$ is further increased to $d_{\mathrm{B}}+4 d_{\mathrm{S}}$ (Fig. 4 (a)), a domain within which the big sphere is stabilized appears additionally around the $x$-axis. The lowest barrier indicated by the thin broken arrow is $\sim k_{\mathrm{B}} T$. The lowest barrier indicated by the thin dotted arrow is considerably lower than $k_{\mathrm{B}} T$ : The big sphere readily reaches the domain around the $x$-axis. The big sphere is confined within the small spaces around the positions indicated by the thick broken and solid arrows where the local potential minima are $\sim-3.5 k_{\mathrm{B}} T$ and $\sim-8 k_{\mathrm{B}} T$ (the potential along the $x$-axis is shown in Fig. 5 (b)), respectively. The small space around the position indicated by the thick solid arrow in 
Fig. 4 (a) is deeper inside the vessel cavity than that in Fig. 3 (a). The big sphere can contact the inner surface of the vessel only with low probability due to high barriers ( $\geq 3 k_{\mathrm{B}} T$ : the case of the lowest barrier is indicated by the thin solid arrow).

For $d_{\mathrm{I}}=d_{\mathrm{B}}+5 d_{\mathrm{S}}$ (Fig. $\left.4(\mathrm{~b})\right)$, there are four domains within which the big sphere is stabilized between the inner surface and the $x$-axis. The lowest barrier indicated by the thin broken arrow is $\sim k_{\mathrm{B}} T$. The lowest barrier indicated by the thin dotted arrow is considerably lower than $k_{\mathrm{B}} T$. The big sphere can be confined within the small spaces around the positions indicated by the thick broken and solid arrows where the local potential minima are $\sim-3 k_{\mathrm{B}} T$ and $\sim-4 k_{\mathrm{B}} T$, respectively. The big sphere can contact the inner surface of the vessel only with low probability due to high barriers ( $\geq 3 k_{\mathrm{B}} T$ : the case of the lowest barrier is indicated by the thin solid arrow). For $d_{\mathrm{I}}=d_{\mathrm{B}}+6 d_{\mathrm{S}}$ (not shown), the small space around the $x$-axis within which the big sphere is confined possesses the local potential minimum $\sim-4 k_{\mathrm{B}} T$.

\subsection{Further discussion}

For $d_{\mathrm{I}}=d_{\mathrm{B}}+n d_{\mathrm{S}}(n=0,1)$, the big-sphere insertion can rarely occur. For $d_{\mathrm{I}}=d_{\mathrm{B}}+n d_{\mathrm{S}}(n \geq 2)$, the big sphere is spontaneously inserted into the vessel. There are $(n-1)$ domains within which the big sphere can be stabilized inside the vessel without contacting the inner surface. For $n=$ even, there is one such domain around the $x$-axis and the big sphere is stabilized the most within it. For $n=$ odd, such domains appear only between the inner surface and the $x$-axis. In general, the stabilization becomes progressively weaker as $n$ increases. The big sphere is confined within a small space. The small space is considerably deep inside for $n \geq 3$. The powers of the insertion and the confinement become progressively weaker as $n$ increases. We find that as $n$ increases further the basic pattern of the entropic potential converges rather rapidly: There are $(n-1)$ small spaces within which the big sphere can be confined around $x / d_{\mathrm{S}^{\sim}}-1$ and their local potential minima are in the range from $\sim-4 k_{\mathrm{B}} T$ to $\sim-3 k_{\mathrm{B}} T$. This is due to the bottom wall of the vessel. When the bottom wall is removed, the potential minima originating from it disappears.

The big sphere is strongly confined (in the sense that the local potential minimum is lower than $-6 k_{\mathrm{B}} T$ corresponding to $\left.g_{12} \sim 400\right)$ within a small space almost in the center of the vessel cavity only for $n=2,3$, and 4 . In the present analysis, $d_{\mathrm{I}}$ is varied with $d_{\mathrm{B}}$ kept constant. However, the behavior exhibited when $d_{\mathrm{B}}$ is varied with $d_{\mathrm{I}}$ kept constant can be conjectured as follows: The strong confinement occurs only when $d_{\mathrm{B}}$ lies in a specific range. Interestingly enough, the big sphere is strongly confined almost in the center, which is in accord with the experimental observation that a protein is in the center of the GroEL-GroES cavity and separated from the inner surface [22]. 
We remark that the quantitative aspects of the results are dependent on the value of $d_{\mathrm{B}}$ adopted. If $d_{\mathrm{B}}$ is set at a larger value (e.g., $d_{\mathrm{B}}=m d_{\mathrm{S}}(m \geq 6)$ and $\left.d_{\mathrm{I}}=d_{\mathrm{B}}+n d_{\mathrm{S}}(n=2,3,4, \ldots)\right)$, the amplitudes of the oscillatory potentials become larger. That is, the big sphere has to overcome higher free-energy barriers for reaching a domain within which it is stabilized. The small space within which the big sphere is confined possesses a lower local potential minimum: The local potential minimum can be lower than $-6 k_{\mathrm{B}} T$ even for $n \geq 5$ when $m$ is sufficiently large. Thus, the range of the big-sphere diameter leading to the strong confinement mentioned in the last paragraph becomes wider as $d_{\mathrm{B}}$ increases.

We discuss Figs. 2 (a), 2 (b), and 3(a). The physical origin of the barriers indicated by the thin arrows is the same as that of the repulsive peak appearing at $h \sim 0.5 d_{\mathrm{S}}$ ( $h$ is the surface separation) in the interaction between big spheres or between a big sphere and a flat wall [1,2,6-11]. The appearance of the red domain in Fig. 2 (b) and that of the blue domain around the $x$-axis inside the vessel in Fig. 3 (a) can be understood in the following way. For the case where the big sphere is at a position on the $x$-axis, the packing of small spheres is illustrated in Fig. 6 . Figure 6 (a) and (b) are prepared for Fig. 2 (b) and Fig. 3 (a), respectively. In Fig. 6 (a), there are two vacant spaces which the small spheres cannot enter and the packing efficiency is considerably low, causing a large entropic loss. By contrast, the small spheres are efficiently packed in Fig. 6 (b). Thus, the entropic effect is remarkably influenced by the packing efficiency of small spheres within a narrow space confined by the big sphere and the inner surface of the vessel. The appearance of the red and blue domains in Figs. 3 (b), 4 (a), and 4 (b) can be understood in a similar way.

For the big sphere to be inserted into the cylindrical vessel, some small spheres have to be removed from the inside of the vessel. When the inner diameter is quite small as in Fig. 2, the small spheres would have a difficult time in going out to the outside while the big sphere is entering. However, in the real system the big sphere and the vessel with polyatomic structures are not rigid but deformable. It is probable that the big sphere and the vessel are deformed so that the small spheres can leave the inside of the vessel.

The water inside a cylindrical vessel could evaporate if the vessel surface is highly hydrophobic. For the evaporation to occur, however, the inner diameter of the vessel must be considerably small. For example, in a molecular dynamics simulation study in which a purely repulsive potential is considered for the surface-water interaction [31], it has been shown that the evaporation occurs only when the surface separation becomes smaller than twice of the molecular diameter of water. Further, the surface-water van der Waals attraction has large effects on the properties of the confined water. When the attraction is taken into account, it is indefinite whether the evaporation actually occurs or not. On the other hand, if the vessel surface is hydrophilic, it is difficult for the big sphere to expel the water from the inside. In the real system, the vessel surface 
possesses both of hydrophobic and hydrophilic patches. As a consequence, the confined water behaves somewhat like the hard-sphere fluid as far as the entropic effect is concerned [2]. Thus, in our opinion the basic physics can be captured by our hard-body model even for the confined system considered in the present study.

\section{Concluding Remarks}

We have calculated the spatial distribution of the entropic potential between a big sphere and an even bigger vessel with cylindrical shape shown in Fig. 1, which are immersed in small spheres forming the solvent, using the three-dimensional integral equation theory. The hard-body models are employed to exclusively investigate the effect of the translational entropy of the solvent. The distribution is strongly dependent on relative magnitudes of the big-sphere diameter $d_{\mathrm{B}}$ and the inner diameter of the vessel $d_{\mathrm{I}}$ unless $d_{\mathrm{I}}$ is much larger than $d_{\mathrm{B}}$. In the present analysis, $d_{\mathrm{I}}$ is varied with $d_{\mathrm{B}}$ kept constant. However, the behavior exhibited when $d_{\mathrm{B}}$ is varied with $d_{\mathrm{I}}$ kept constant can also be conjectured: A big sphere whose diameter lies in a specific range is not only spontaneously inserted into the vessel but also strongly confined within a small space almost in the center of the vessel cavity. Moreover, the specific range becomes wider as $d_{\mathrm{B}}$ increases.

The insertion of a big body into a big vessel is an important process in biological systems. Typical examples are the introduction of a polypeptide into the chaperonin GroEL $[21,22]$ and that of an antibiotic molecule or a toxic protein into a cell-membrane protein [23,24]. In these processes, the entropic effect arising from the translational displacement of solvent molecules should play crucially important roles. In the case of the chaperonin GroEL, $d_{\mathrm{B}} / d_{\mathrm{S}}$ is considerably larger than 5 , the value considered in the present calculations. We note that the entropic effect becomes stronger with increasing $d_{\mathrm{B}} / d_{\mathrm{S}}$ [2]. The electrostatic interaction, which would be quite strong in pure water, is largely screened by the counter ions in aqueous solution under the physiological condition containing $\sim 0.15 \mathrm{M} \mathrm{NaCl}$. As a consequence, the entropic effect is expected to be dominantly large in comparison with the other effects.

Effects of the tapering of $d_{\mathrm{I}}$ along $-x$ or $+x$ direction for the vessel and the asphericity of the big body are to be investigated in the next step. The exclusion of the big body, which was already inserted into the vessel, to the outside could also be elucidated by the entropic effect: It could be achieved by changing the geometric features of the vessel and/or the big body after the insertion of the big body is completed. The details of the polyatomic structures of the vessel and/or the big body are important factors. As for the dynamical aspects of the system behavior, a rigorous method is to simulate a biased Brownian motion of a big sphere in an entropic force field 
calculated in the present study. These are interesting subjects to be pursued as future works.

\section{Acknowledgment}

This work was supported by Grants-in-Aid for Scientific Research on Innovative Areas (No. 20118004) from the Ministry of Education, Culture, Sports, Science and Technology of Japan, by the Grand Challenges in Next-Generation Integrated Nanoscience, MEXT, Japan, and by Kyoto University Global Center of Excellence (GCOE) of Energy Science.

\section{References}

[1] M. Kinoshita, Chem. Eng. Sci. 61 (2006) 2150.

[2] M. Kinoshita, Front. Biosci. 14 (2009) 3419.

[3] M. Kinoshita, Int. J. Mol. Sci. 10 (2009) 1064.

[4] S. Asakura and F. Oosawa, J. Chem. Phys. 22 (1954) 1255.

[5] S. Asakura and F. Oosawa, J. Polymer Sci. 33 (1958) 183.

[6] P. Attard and G. N. Patey, J. Chem. Phys. 92 (1990) 4970.

[7] T. Biben, P. Bladon, and D. Frenkel, J. Phys. Condens. Matter 8 (1996) 10799.

[8] M. Kinoshita, S. Iba, K. Kuwamoto, and M. Harada, J. Chem. Phys. 105 (1996) 7177.

[9] R. Dickman, P. Attard, and V. Simonian, J. Chem. Phys. 107 (1997) 205.

[10] B. Götzelmann, R. Evans, and S. Dietrich, Phys. Rev. E 57 (1998) 6785.

[11] R. Roth, R. Evans, and S. Dietrich, Phys. Rev. E 62 (2000) 5360.

[12] R. Roth, B. Götzelmann, and S. Dietrich, Phys. Rev. Lett. 83 (1999) 448.

[13] M. Kinoshita and T. Oguni, Chem. Phys. Lett. 351 (2002) 79.

[14] M. Kinoshita, J. Chem. Phys. 116 (2002) 3493.

[15] P. Bryk, R. Roth, M. Schoen, and S. Dietrich, Europhys. Lett. 63 (2003) 233.

[16] R. Roth, R. van Roji, D. Andrienko, K. R. Mecke, and S. Dietrich, Phys. Rev. Lett. 89 (2002) 088301 .

[17] M. Kinoshita, Chem. Phys. Lett. 387 (2004) 47.

[18] M. Kinoshita, Chem. Phys. Lett. 387 (2004) 54.

[19] P.-M. König, R. Roth, and S. Dietrich, Europhys. Lett. 84 (2008) 68006.

[20] R. Roth and M. Kinoshita, J. Chem. Phys. 125 (2006) 084910.

[21] A. L. Horwich, W. A. Fenton, E. Chapman, and G. W. Farr, Annu. Rev. Cell. Dev. Biol. 23 
(2007) 115.

[22] R. Kanno, A. Koike-Takeshita, K. Yokoyama, H. Taguchi, and K. Mitsuoka, Structure 17 (2009) 287.

[23] V. Koronakis, A. Sharff, E. Koronakis, B. Luisi, and C. Hughes, Nature 405 (2000) 914.

[24] L. Federici, F. Walas, and B. Luisi, Curr. Sci. 87 (2004) 190.

[25] D. Beglov and B. Roux, J. Chem. Phys. 103 (1995) 360.

[26] M. Ikeguchi and J. Doi, J. Chem. Phys. 103 (1995) 5011.

[27] Y. Harano and M. Kinoshita, Chem. Phys. Lett. 399 (2004) 342.

[28] Y. Harano and M. Kinoshita, Biophys. J. 89 (2005) 2701.

[29] R. Roth, Y. Harano, and M. Kinoshita, Phys. Rev. Lett. 97 (2006) 078101.

[30] J. C. Crocker, J. A. Matteo, A. D. Dinsmore, and A. G. Yodh, Phys. Rev. Lett. 82 (1999) 4352. [31] A. Wallqvist and B. J. Berne, J. Phys. Chem. 99 (1995) 2893. 


\section{Figure Captions}

Fig. 1. (a) Big vessel considered. It is colored for three-dimensional visualization. (b) Its cross section magnified $(z=0)$. The thick solid lines represent the $x$-axis and $y$-axis. $L, d_{\mathrm{O}}, d_{\mathrm{I}}$, and $D$ are the length, outer and inner diameters, and thickness of the bottom wall, respectively. $L,\left(d_{\mathrm{O}}-d_{\mathrm{I}}\right) / 2$, and $D$ are fixed at $7 d_{\mathrm{S}}, 6 d_{\mathrm{S}}$, and $d_{\mathrm{S}}$, respectively, and $d_{\mathrm{I}}$ is varied as an important parameter. The numbers given are scaled by $d_{\mathrm{S}}$. When the big-sphere diameter $d_{\mathrm{B}}$ is set at $5 d_{\mathrm{S}}$, the center of the big sphere in contact with the bottom wall is at $(x, y)=(0,0)$.

Fig. 2. Spatial distribution of entropic potential scaled by $k_{\mathrm{B}} T$ between the big sphere and the vessel for different values of $d_{\mathrm{I}}$ : (a) $d_{\mathrm{I}}=d_{\mathrm{B}}$ and (b) $d_{\mathrm{I}}=d_{\mathrm{B}}+d_{\mathrm{S}}$. As the color approaches thick blue, the potential becomes lower, and as the color approaches thick red, the potential becomes higher. The center of the big sphere cannot enter the domain drawn in white. The numbers given are scaled by $k_{\mathrm{B}} T$. The maximum and minimum values of the potential scaled by $k_{\mathrm{B}} T$ are also given.

Fig. 3. Spatial distribution of entropic potential scaled by $k_{\mathrm{B}} T$ between the big sphere and the vessel for different values of $d_{\mathrm{I}}$ : (a) $d_{\mathrm{I}}=d_{\mathrm{B}}+2 d_{\mathrm{S}}$ and (b) $d_{\mathrm{I}}=d_{\mathrm{B}}+3 d_{\mathrm{S}}$. As the color approaches thick blue, the potential becomes lower, and as the color approaches thick red, the potential becomes higher. The center of the big sphere cannot enter the domain drawn in white. The numbers given are scaled by $k_{\mathrm{B}} T$. The maximum and minimum values of the potential scaled by $k_{\mathrm{B}} T$ are also given.

Fig. 4. Spatial distribution of entropic potential scaled by $k_{\mathrm{B}} T$ between the big sphere and the vessel for different values of $d_{\mathrm{I}}$ : (a) $d_{\mathrm{I}}=d_{\mathrm{B}}+4 d_{\mathrm{S}}$ and (b) $d_{\mathrm{I}}=d_{\mathrm{B}}+5 d_{\mathrm{S}}$. As the color approaches thick blue, the potential becomes lower, and as the color approaches thick red, the potential becomes higher. The center of the big sphere cannot enter the domain drawn in white. The numbers given are scaled by $k_{\mathrm{B}} T$. The maximum and minimum values of the potential scaled by $k_{\mathrm{B}} T$ are also given.

Fig. 5. Entropic potential along the $x$-axis scaled by $k_{\mathrm{B}} T$ between the big sphere and the vessel for different values of $d_{\mathrm{I}}$ : (a) $d_{\mathrm{I}}=d_{\mathrm{B}}+2 d_{\mathrm{S}}$ and (b) $d_{\mathrm{I}}=d_{\mathrm{B}}+4 d_{\mathrm{S}}$.

Fig. 6. Packing of small spheres within the narrow space confined by the big sphere and the inner surface of the vessel for different values of $d_{\mathrm{I}}$ : (a) $d_{\mathrm{I}}=d_{\mathrm{B}}+d_{\mathrm{S}}$ and (b) $d_{\mathrm{I}}=d_{\mathrm{B}}+2 d_{\mathrm{S}}$. 


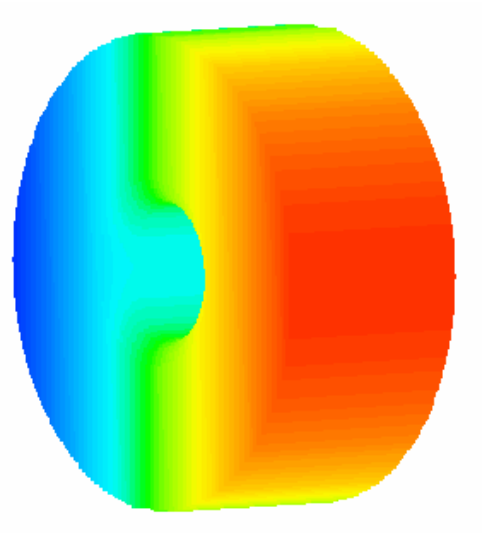

(a)

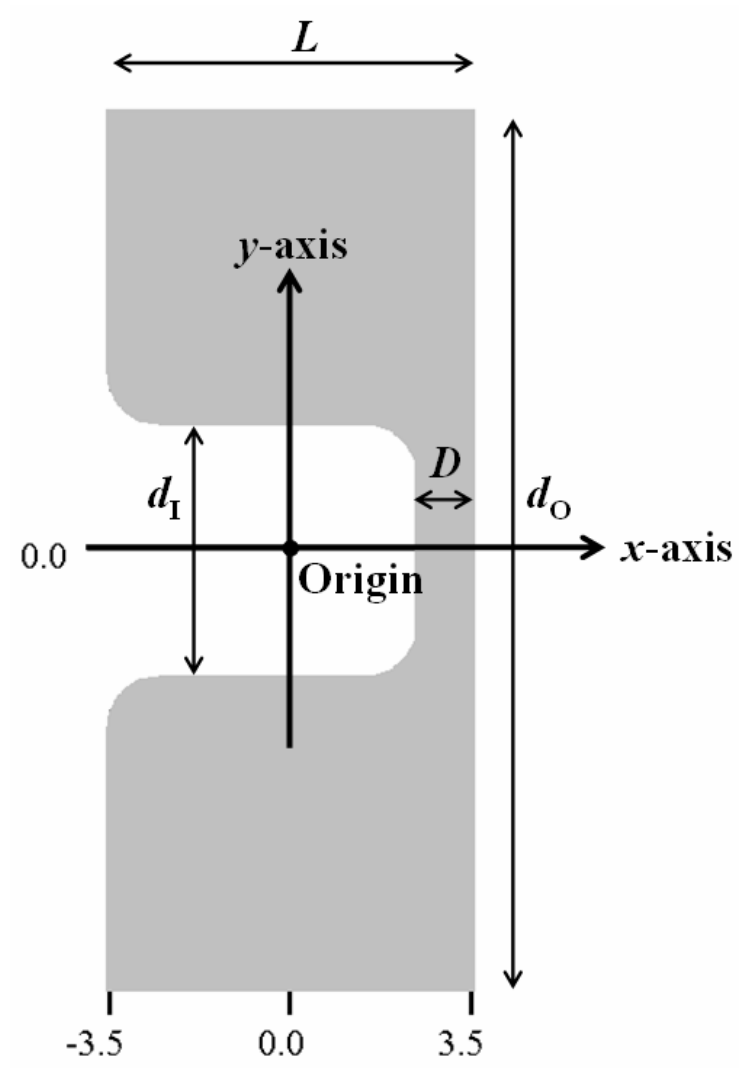

(b)

Figure 1 


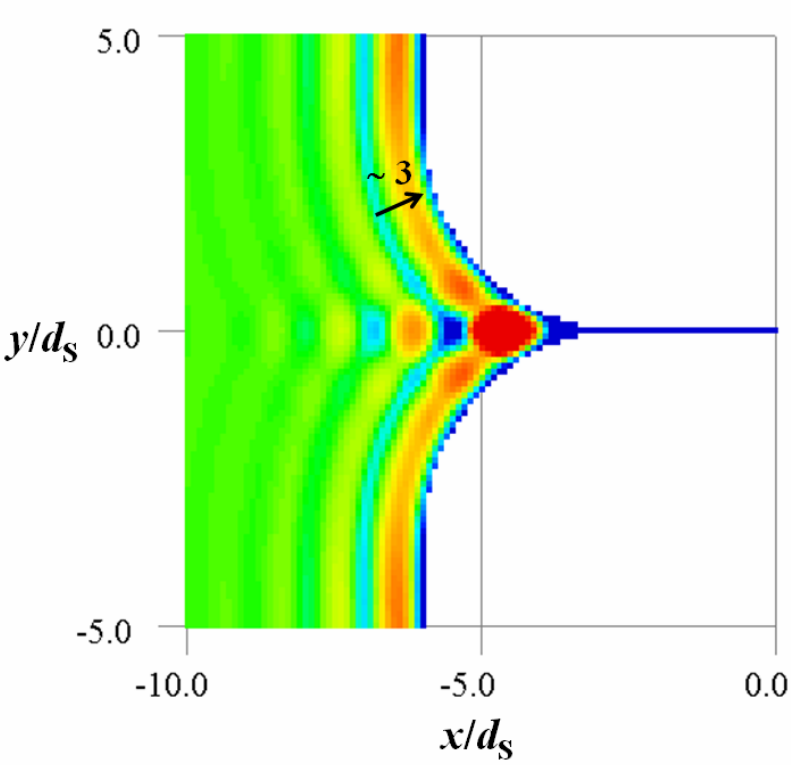

Max: 10.6

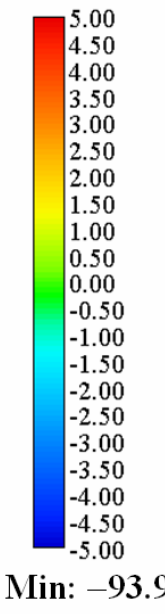

(a)

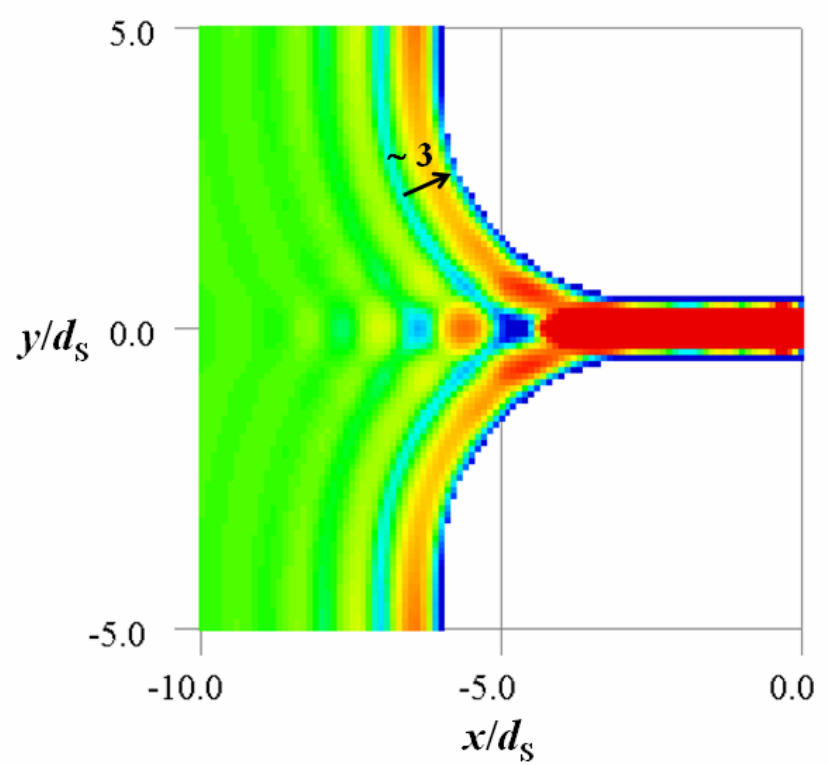

Max: 27.8

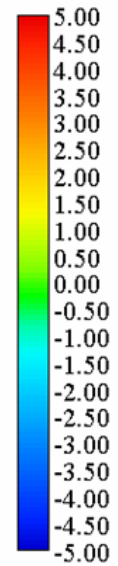

Min: -18.2

(b) 


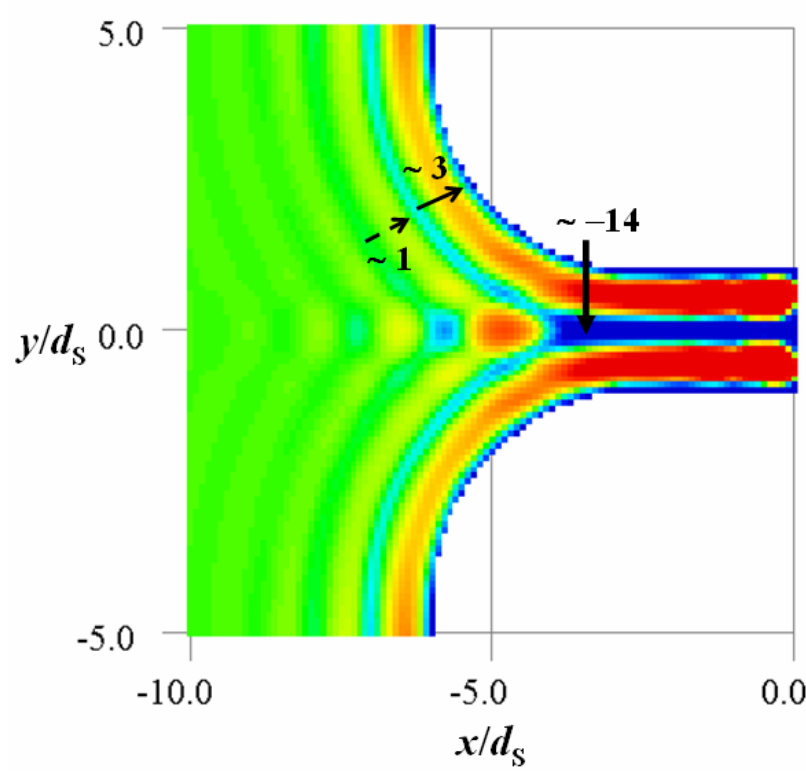

Max: 14.1

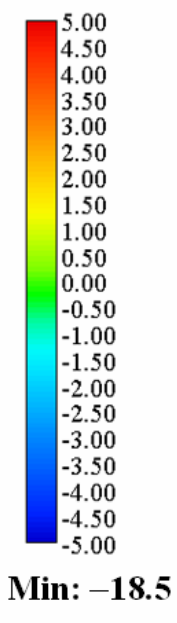

(a)

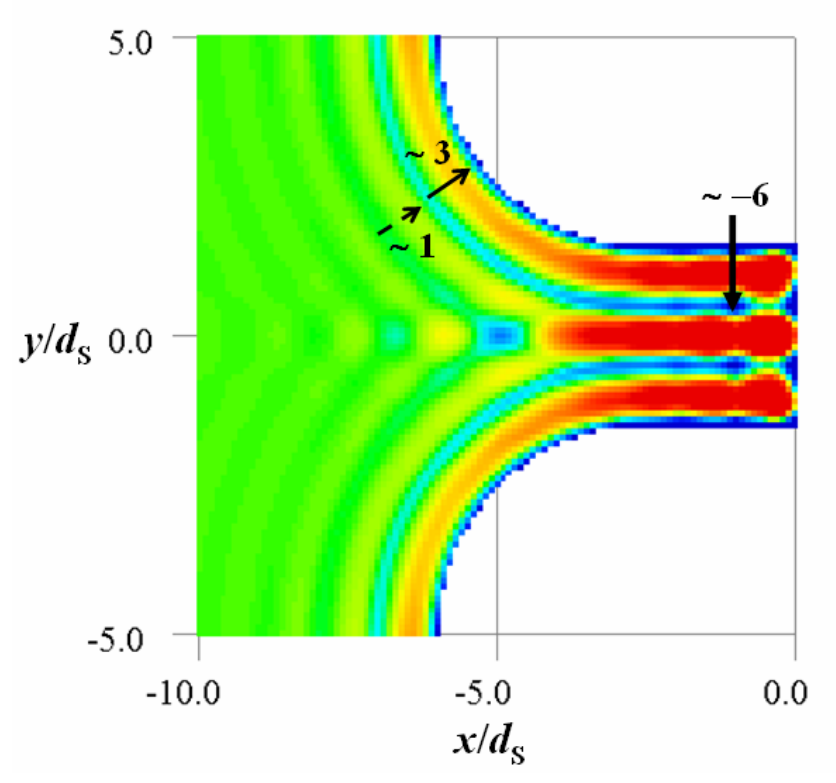

Max: 13.8

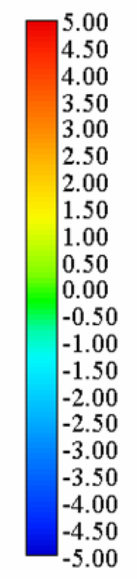

Min: -16.4

(b)

Figure 3 


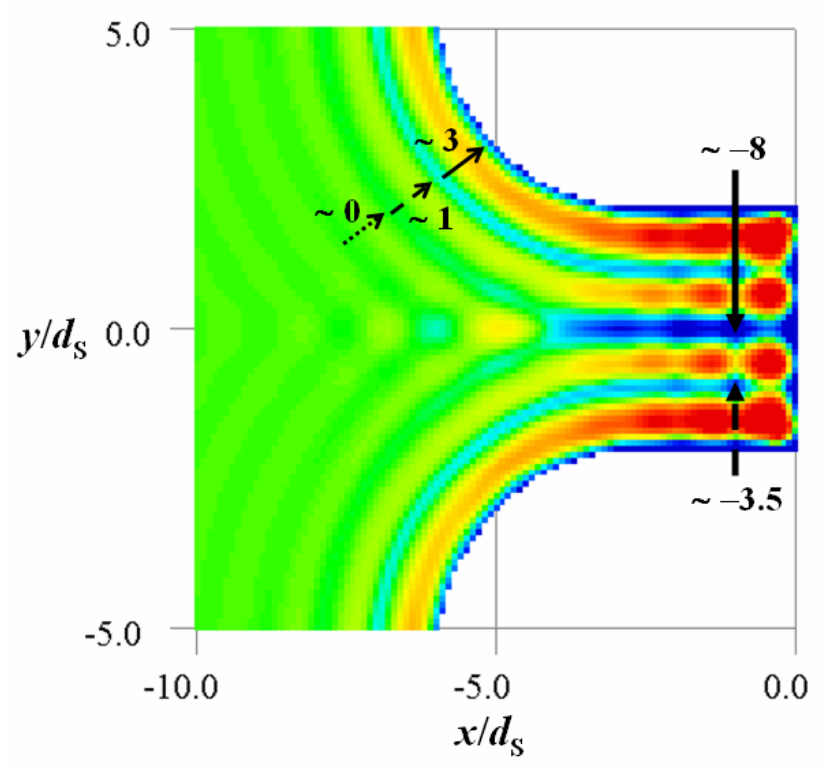

Max: 8.87

(a)

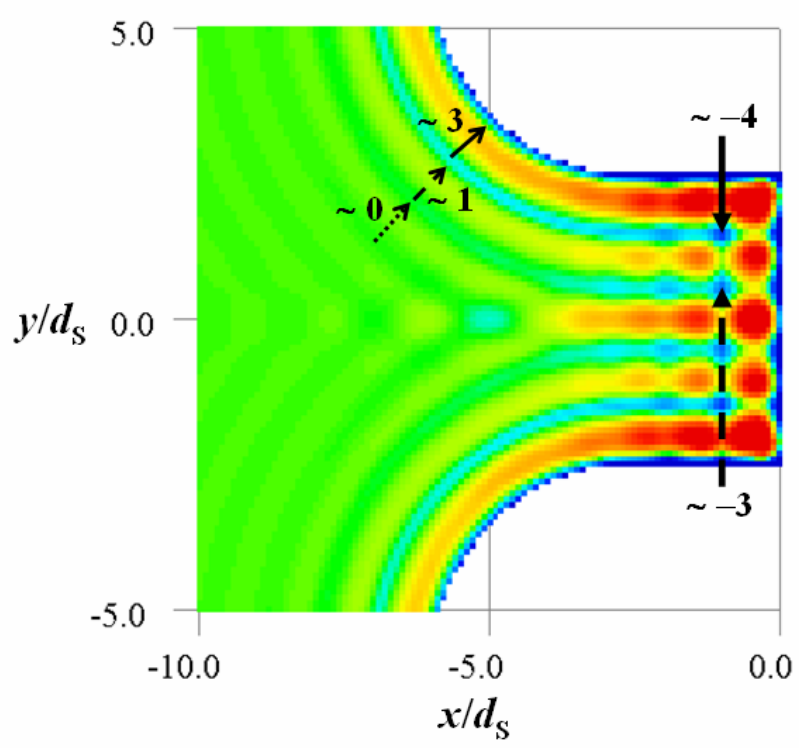

Max: 8.38

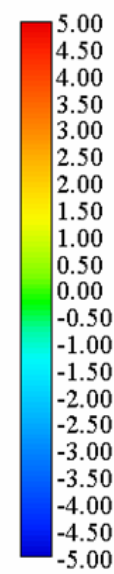

Min: -15.94

(b) 


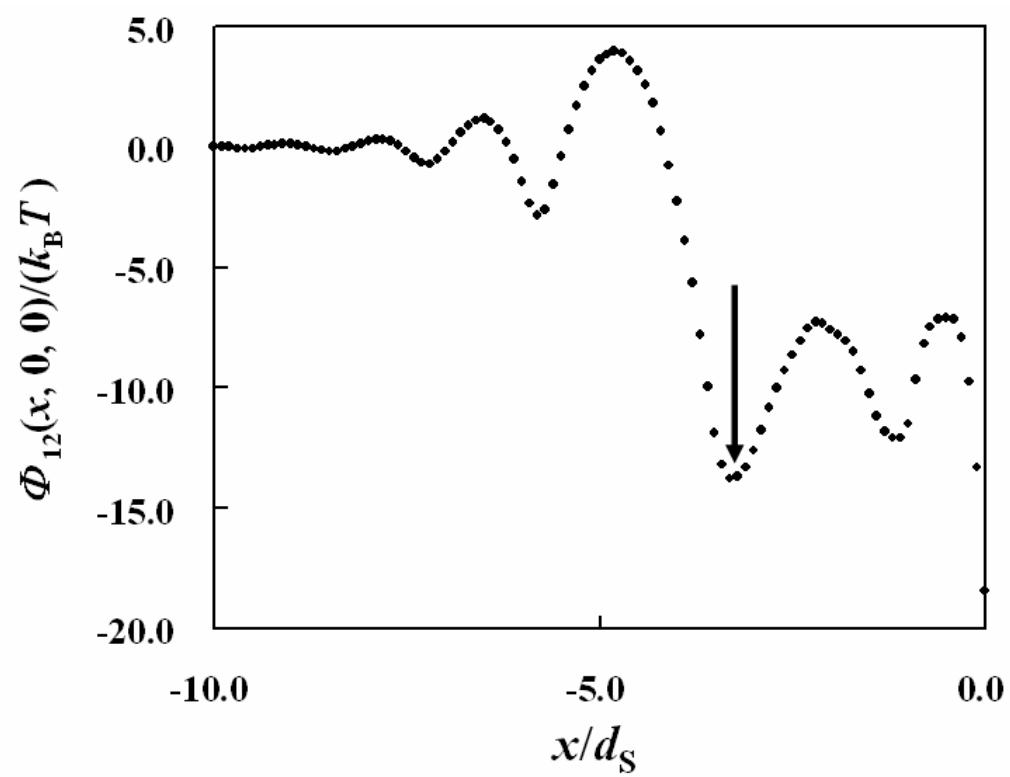

(a)

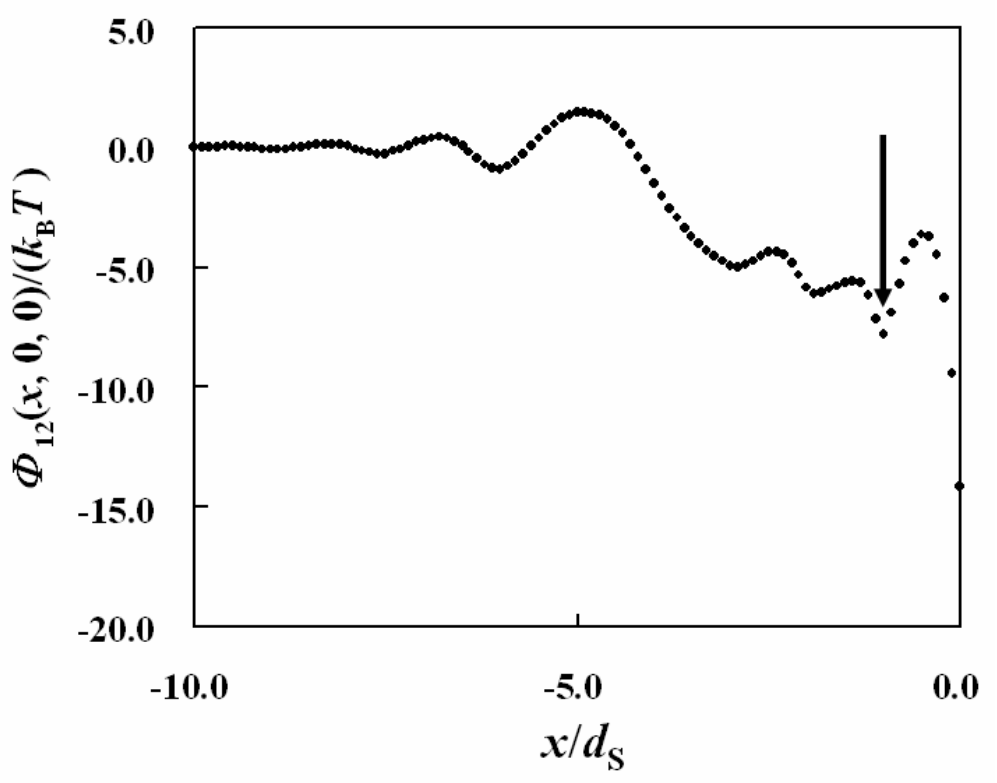

(b) 


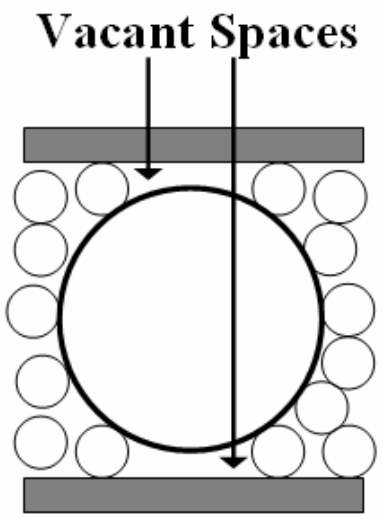

(a)

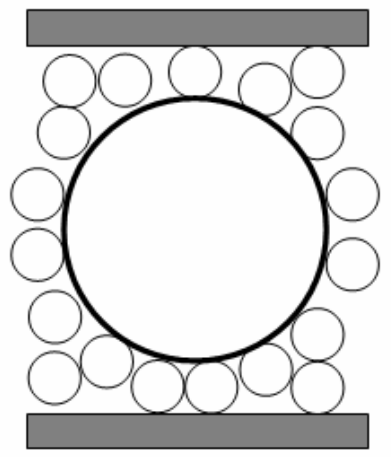

(b)

Figure 6 